\title{
The value of Gd-BOPTA- enhanced MRIs and DWI in the diagnosis of intrahepatic mass-forming cholangiocarcinoma
}

\author{
C. C. XU' ${ }^{1, *}$, Y. F. TANG ${ }^{1}$, X. Z. RUAN ${ }^{1}$, Q. L. HUANG ${ }^{1}$, L. SUN ${ }^{2}$, J. $\mathrm{LI}^{2}$ \\ ${ }^{1}$ Department of Imaging, Ningbo First Hospital, Ningbo 315010, P.R. China; ${ }^{2}$ Department of Imaging, Wenzhou Central Hospital, Wenzhou \\ 325000, P.R. China \\ *Correspondence: Dr_xcc@163.com
}

Received November 25, 2016 / Accepted April 20, 2017

\begin{abstract}
The aim of this study is to explore the value of unenhanced magnetic resonance imaging (MRI), gadobenate dimeglumine injection (Gd-BOPTA)-enhanced MRI and diffusion-weighted imaging (DWI) in the diagnosis of intrahepatic mass-forming cholangiocarcinoma (IMCC). Totally 59 IMCC patients who underwent Gd-BOPTA-enhanced MRIs were recruited. The time-signal intensity curves and lesion periphery enhancement rates of the IMCC and liver parenchyma was drawn using apparent diffusion coefficient (ADC) values. The Gd-BOPTA-enhanced MRI showed that the peripheries of 30 lesions in the arterial phase exhibited irregular ring enhancement. However, lesions in the portal and delayed phases (which were gradually filled with a contrast agent), presented a patchy or latticed enhancement. Twenty-two lesions in the arterial and delayed phases exhibited uneven mild/moderate patchy enhancements with a progressive and centripetal lesion. Five lesions emerged from the arterial phase without any significant enhancement and had only gradual enhancement during the delayed phase. The remaining 2 lesions had a decreased mild enhancement, presented comparatively high signals and the lesion center had visible small spotted low signals. The DWI signals displayed a slightly high or high unevenness. Some lesion peripheries had a high signal but lesion centers displayed a relatively low or slightly low signal and irregular patches. There were significant differences between the ADC values of the lesion edge, lesion center and liver parenchyma. The IMCC detection rates of the Gd-BOPTA-enhanced MRI and DWI were higher than those of the unenhanced MRI. Our study demonstrated that both the Gd-BOPTA-enhanced MRI and DWI had higher accuracies rates than an unenhanced MRI. Furthermore, the hepatobiliary phase of IMCC plays an important role in the diagnosis and identification of IMCC constituents.
\end{abstract}

Key words: intrahepatic mass-forming cholangiocarcinoma, magnetic resonance imaging, dynamic contrast-enhanced magnetic resonance imaging, gadobenate dimeglumine, diffusion weighted imaging, enhancement rate

Intrahepatic cholangiocarcinoma (ICC) is a primary hepatic adenocarcinoma that originates from the intrahepatic biliary tract epithelium [1]. ICC is the second most common liver malignancy behind hepatocellular cancer and accounts for approximately $15 \%$ to $20 \%$ of all primary liver cancers [2]. Based on the macroscopic appearance, ICC can be separated into three types: mass forming, intraductal growing and periductal infiltrating [3]. Intrahepatic mass-forming cholangiocarcinoma (IMCC) has a radial growth pattern, constitutes about $60 \%$ of all ICC and can invade the liver parenchyma [2]. Several typical risk factors of ICC include primary sclerosing cholangitis, hepatolithiasis, choledochal cysts, parasitic infestation of the bile tract and chronic liver diseases such as chronic hepatitis B and hepatitis C [4]. The optimal treatment option for ICC is hepatic resection. However, patients with this disease are commonly surgically un-operable due to the presence of multiple intrahepatic and lymph node metastasis, peritoneal dissemination and/or extrahepatic distant metastasis. The 5-year survival rate ranges from $4.1 \%$ to $43.8 \%$ [5, 6]. Recently, imaging modalities such as magnetic resonance imaging (MRI), multidetector computed tomography (CT), ultrasonography and positron emission tomography/CT have been adopted, either alone or in combination, for the diagnosis and treatment planning of IMCC [7].

Magnetic resonance imaging (MRI) is widely utilized for image evaluation of the liver as it is able to show intrinsic soft tissue contrasts [8]. The application of a conventional MRI conducted without contrast or non-specific agents like extracellular gadolinium chelates can show variety of liver specific contrasts, improve the morphological evaluation and provide 
very useful information [9]. Among these non-specific agents, gadolinium-based hepatobiliary agents are superior for the examination and characterization of hepatic focal lesions. Two gadolinium-based hepatobiliary agents are widely utilized and have been clinically approved: gadolinium ethoxybenzyl diethylenetriaminepentaacetic acid (gadoxetic acid disodium or Gd-EOB-DTPA) and gadobenate dimeglumine (Gd-BOPTA). Gd-BOPTA received Food and Drug Administration (FDA) approval in 2004. It has the dual properties of being a hepatobiliary contrast agent and an extracellular agent. Increasing evidence demonstrates that the use of Gd-BOPTA achieves better vascular enhancement than Gd-EOB-DTPA [10]. Currently, most clinical research only investigate the diagnostic significance of Gd-BOPTA-enhanced MRIs and Gd-EOBDTPA-enhanced MRIs in patients with liver diseases. Few studies have been performed to compare unenhanced MRIs, Gd-BOPTA-enhanced MRIs and diffusion-weighted imaging (DWI) in relation to liver diseases [11, 12]. Additionally, no reliable radiological characteristics are available to distinguish IMCs from metastatic adenocarcinomas. Therefore, the diagnosis of IMCC excludes any extrahepatic primary tumors. The purpose of this study is to assess and compare the diagnostic significance of unenhanced MRIs, Gd-BOPTA-enhanced MRIs and DWI in IMCC.

\section{Materials and methods}

Ethics statement. This study was approved by the ethics committee of Ningbo First hospital. Written informed consent was obtained from each study subject and/or their legal guardians. This study complies with the guidelines and principles of the declaration of Helsinki [13].

Subjects. A total of 59 patients with IMCC who underwent Gd-BOPTA-enhanced MRI dynamic multi-phase scanning at the radiology department at Ningbo First hospital between March 2014 and March 2015 were recruited. The exclusion criteria were as following: (1) patients younger than 18 years of age; (2) pregnant or lactating women; (3) patients with general contraindications indicated through MRI examination; (4) patients without complete imaging data or a pathological diagnosis.

Instruments and method. A 3T MRI scanner (Magnetom Trio, Magnetom Verio; Siemens Medical Solutions, Erlangen, Germany) was utilized for this experiment. Body coils were used as the transmitting coil and pelvic phased array coils were used as the receiving coil. The partial sequence scan was conducted while patients held their breath. In preparation for the scan, patients fasted for 4 to 6 hours to avoid motion artifact and also received breathing and breath-holding training. In addition, patients who lay in a supine head first position received both unenhanced and dynamic contrastenhanced MRIs.

The scanning sequence of the unenhanced MRI was: (1) axial turbo spin echo (TSE) T1-weighted imaging (T1WI) $($ repetition time $(\mathrm{TR})=550 \mathrm{~ms}$; echo time $(\mathrm{TE})=14 \mathrm{~ms}$; ma- trix $=320 \times 216$; field of view $(\mathrm{FOV})=342 \times 308$; bandwidth $=256 \mathrm{~Hz}$; thickness $=6 \mathrm{~mm}$; interlamellar spacing $=1.2 \mathrm{~mm}$ ); (2) axial fat suppressed TSE T2-weighted imaging (T2WI) $(\mathrm{TR}=3000 \mathrm{~ms} ; \mathrm{TE}=104 \mathrm{~ms} ;$ matrix $=168 \times 320 ; \mathrm{FOV}=285$ $\times 380$; bandwidth $=260 \mathrm{~Hz}$; thickness $=5 \mathrm{~mm}$; interlamellar spacing $=1 \mathrm{~mm}) ;(3)$ coronal TSE T2WI $(\mathrm{TR}=1400 \mathrm{~ms}$; TE $=93 \mathrm{~ms}$; matrix $=224 \mathrm{p} \times 320 ; \mathrm{FOV}=380 \times 380$; bandwidth $=446 \mathrm{~Hz}$; thickness $=5 \mathrm{~mm}$; interlamellar spacing $=1 \mathrm{~mm}$ ); (4) in-and-out-of-phase gradient recalled echo (GRE) T1WI (in phase: $\mathrm{TR}=130 \mathrm{~ms}$; $\mathrm{TE}=2.3 \mathrm{~ms}$; matrix $=154 \times 256$; FOV $=285 \times 380$; bandwidth $=930 \mathrm{~Hz}$; thickness $=5 \mathrm{~mm}$; interlamellar spacing $=1 \mathrm{~mm}$ ); out-of-phase: out of phase TR $=130 \mathrm{~ms} ; \mathrm{TE}=3.7 \mathrm{~ms} ;$ matrix $=154 \times 256 ; \mathrm{FOV}=285 \times 380$; bandwidth $=977 \mathrm{~Hz}$; thickness $=5 \mathrm{~mm}$; interlamellar spacing = $1 \mathrm{~mm}$ ); (5) DWI with respiratory triggered single-shot spin-echo echo-planar imaging (SS SE-EPI) sequence plus fat suppression technology $(\mathrm{TR}=3636.8 \mathrm{~ms} ; \mathrm{TE}=73 \mathrm{~ms}$; matrix $=78 \times 128 ; \mathrm{FOV}=285 \times 380$; thickness $=5 \mathrm{~mm}$; interlaellar spacing $=1 \mathrm{~mm}$; five b values: $50,100,500,800,1200 \mathrm{~s} / \mathrm{mm}^{2}$, multilayer images were obtained during one single breath); (6) thick slab two-dimensional magnetic resonance cholangiopancreatography $(\mathrm{MRCP})$ in the coronal plane $(\mathrm{TR}=4500$ $\mathrm{ms}$; TE $=735 \mathrm{~ms}$; bandwidth $=352 \mathrm{~Hz}$; matrix $=269 \times 384$; FOV $=300 \times 380$; thickness $=50 \mathrm{~mm}$ ) and 4 to 8 multi-angle images were collected.

A three-dimensional volume interpolated body examination (VIBE) was utilized for the pre-scan process before a dynamic contrast-enhanced MRI was performed $(\mathrm{TR}=3.9$ $\mathrm{ms}$; $\mathrm{TE}=1.4 \mathrm{~ms}$; bandwidth $=352 \mathrm{~Hz}$; matrix $=182 \times 320$; FOV $=309 \times 251$; bandwidth $=401 \mathrm{~Hz}$; thickness $=3.0 \mathrm{~mm}$; interlamellar spacing $=0.6 \mathrm{~mm}$; number of excitation $(\mathrm{NEX})=$ 2 ; imaging time $=17 \mathrm{~s}$ ). After the pre-scan, patients were given cubital vein injections of Gd-BOPTA (MultiHance, Shanghai Braccosine Pharmaceutical Co., Ltd.) through an automatic double-syringe power injector (ULRICH, Germany). The dose of MultiHance was $0.2 \mathrm{ml} / \mathrm{kg}$ and was injected at a constant rate of $2.5 \mathrm{~mL} / \mathrm{s}$. The MRI scanning began with the same conditions of the pre-scan from the early arterial phase (15 s). Next, dynamic contrast-enhanced images were collected during MRI scanning in the late arterial phase (25 s), portal phase (59.5 $\mathrm{s} \pm$ $3.7 \mathrm{~s}$ ), equilibrium phase ( $1 \mathrm{~min} 40.5 \mathrm{~s} \pm 9 \mathrm{~s}$ ), delayed phase (5 $\mathrm{min} 10.8 \mathrm{~s} \pm 3 \mathrm{~min}$ ) and hepatobiliary phase (60 $\mathrm{min} 40 \mathrm{~s} \pm$ $5 \min 24 s$ and $97 \min 30 s \pm 15 \min 9 s$ ).

Image analysis. The MRI data of all patients was analyzed and compared to the pathological results. Two sets of results were obtained by two physicians experienced in abdominal imaging diagnosis and consensus was reached. The image analysis included: (1) the image features of IMCC in the hepatobiliary phase from the dynamic contrast-enhanced MRI; (2) the time-signal intensity curves and lesion periphery, center of IMCC and liver parenchyma tendencies; (3) the dynamic contrast-enhanced MRI features from the arterial to delayed phases and the IMCC related signs such as cholangiectasis, liver capsule shrinkage, liver atrophy, vascular involvement, invasion and metastasis of IMCC, lymph node 
of hepatoduodenal ligament and retroperitoneum from the unenhanced MRI.

Time-signal intensity curve. The maximum lesion level was selected and the lesion periphery signal intensity at each phase was calculated by averaging the signal intensities of the three regions of interest (ROI). In the same way, the lesion center signal intensity at each phase was measured and the ROI selection avoided mucus and calcification areas. Three ROIs in the liver lobe (a lesion which avoided blood vessels and the bile duct expansion from each phase) were also selected. The liver parenchyma signal intensity of each phase was also calculated by averaging the signal intensity of the three ROIs. Finally, time-signal intensity curves and lesion periphery, center of IMCC and liver parenchyma tendencies were drawn. The main curve parameters were: the area's baseline $\left(\mathrm{SI}_{0}\right)$, maximal signal intensities $\left(\mathrm{SI}_{\max }\right)$, maximal time $\left(\mathrm{T}_{\max }\right)$ and enhancement ratio. These were calculated using the equation: $\mathrm{R}=\left(\mathrm{SI}_{\max }-\mathrm{SI}_{0}\right) / \mathrm{T}_{\max }$. The lesion center, enhancement rate and corresponding times were calculated when the signal intensity reached or was greater than $90 \%$ SI.

Measurement of the apparent diffusion coefficient (ADC) value. The MRI images were transmitted to the clinical medical image information system (Angelwin Technology Co, Ltd, Beijing, China) and ADC images were automatically reconstructed through diffusion weighted imaging (DWI). By collecting dynamically enhanced data, ADC images which corresponded to different $b$ values in the early enhancement artery phase and the delayed enhancement of lesion center and normal liver tissues were able to be measured to indicate the degree of diffusion. From the ADC images, a ROI was drawn in the layer with the largest lesion diameter. The ROI included the maximum lesion area and avoided necrosis as much as possible. Three ROIs were obtained and three tests were performed on each lesion to get an average. If a patient had several lesions the most typical one was selected. ADC values which corresponded to the $b$ values of each ROI were measured.

Statistical analysis. The SPSS 19.0 software (IBM, 2010, Chicago, IL, USA) was used for data analysis. Categorical data is displayed as mean \pm standard deviation (SD) and was tested for a normal distribution. Two groups were compared using the $t$ test. After a homogeneity test of variance, multiple groups were compared using one-way analysis of variance. The two pair comparison of means from multiple groups was performed using the least significant difference (LSD)- $t$ test. All tests were two sided and the level of significance was set at 0.05 .

\section{Results}

The basic information of IMCC patients. A total of 59 IMCC patients, 35 males and 24 females with ages ranging from 44 to 75 years old and a mean age of $61.3 \pm 12.1$ years old were pathologically diagnosed (by surgery $(\mathrm{n}=45)$ and biopsy $(\mathrm{n}=14))$. The clinical symptoms included upper abdominal pain $(n=39)$ fever $(n=3)$, jaundice $(n=7)$, fatigue and loss of appetite ( $n=7)$, focal liver by B ultrasonic $(n=6)$, history of hepatitis B or hepatocirrhosis $(n=6)$, history of Caroli disease $(\mathrm{n}=1)$, intra-and extrahepatic bile duct stone $(\mathrm{n}=$ $20)$ and cholecystectomy $(n=5)$. The laboratory inspection demonstrated the following results. Hepatitis $B$ surface antigen (HBsAg)-positive $(n=19)$, increased white blood cell count $(\mathrm{WBC})(\mathrm{n}=15)$, increased alpha fetoprotein (AFP) $(\mathrm{n}=12)$ of which 4 had mixed hepatocellular cholangiocarcinoma, increased carcino-embryonic antigen (CEA) $(\mathrm{n}=18)$, increased cancer antigen 125 (CA125) $(\mathrm{n}=19)$ and increased CA19-9 $(\mathrm{n}=32)$ of which $23 \mathrm{had}$ greater than $100 \mathrm{U} / \mathrm{ml}$ increases (Table 1).

Lesion location and morphological characteristics. Among the 59 patients, 55 had single lesions, 3 had satellite lesions and 1 had hepatocellular carcinoma (which refers to a large mass in the right hepatic lobe). Cholangiocarcinoma refers to the round nodules in the upper section of the left lateral lobe. Out of the 59 patients, 24 had lesions located in the right hepatic lobe, 31 in the left hepatic lobe and 4 in both lobes. Most of the tumors displayed an irregular or round shape and had diameters ranging from $1.2 \mathrm{~cm}$ to $11.8 \mathrm{~cm}$ (mean: $5.91 \mathrm{~cm} \pm 3.17 \mathrm{~cm}$ ). 32 patients had tumors with a diameter which exceeded $5 \mathrm{~cm}$. Satellite lesions around the

Table 1. The clinical characteristics of the patients with IMCC

\begin{tabular}{lc}
\hline Clinical characteristics & Case (n) \\
\hline Diagnostic methods & 45 \\
Surgery & 14 \\
Biopsy & \\
Gender & 35 \\
Male & 24 \\
Female & $61.3 \pm 12.1$ \\
Mean age (years old) & \\
Clinical symptoms & 39 \\
Upper abdominal pain & 3 \\
Fever & 7 \\
Jaundice & 7 \\
Fatigue and loss of appetite & \\
Abnormal liver function & 6 \\
focal liver & 6 \\
Hepatitis B or hepatocirrhosis & 1 \\
History of Caroli disease & 20 \\
Intra-and extrahepatic bile duct stone & 5 \\
Cholecystectomy & \\
Laboratory inspection & 19 \\
HBsAg-positive & 15 \\
Increased WBC & 12 \\
Increased AFP & 18 \\
Increased CEA & \\
Increased CA125 & \\
Increased CA19-9 & \\
\hline &
\end{tabular}

Note: IMCC, intrahepatic mass-forming cholangiocarcinoma; HBsAg, hepatitis B surface antigen; WBC, white blood cell count; AFP, alpha fetoprotein; CEA, carcino-embryonic antigen; CA125, cancer antigen 125 

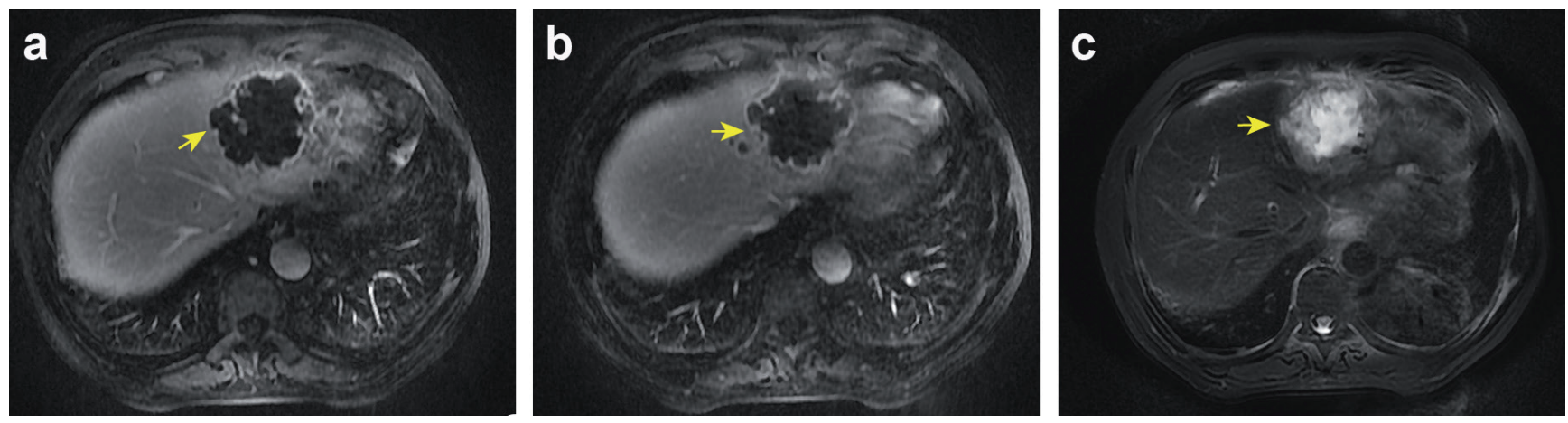

Figure 1. Lesion was located in the left internal and lateral lobe of liver. a: higher signal in small spotted and patchy area on T2WI; b: high signal on DWI ( $b=1200)$; $c$ : decreased signal on ADC image; DWI, diffusion-weighted imaging; ADC, apparent diffusion coefficient.

tumors mainly had a small round shape and diameters ranging from $0.5 \mathrm{~cm}$ to $2 \mathrm{~cm} .40$ patients had lesions which were irregularly infiltrated with a blur periphery. 5 of these lesions invaded the gallbladder and were located either next to the gallbladder fossa in the right anterior lobe or the junction between the right and left lobes. Another 2 lesions invaded the adjacent right adrenal which is located in the right posterior lobe. Lesions of the remaining 19 patients had clear peripheries ( 16 were shallow lobulated and 3 were round).

Symptoms on the unenhanced MRI. The tumor lesions of 41 patients showed a slight signal unevenness and strong T2 signals. Twenty-two of these lesions had "soft vine" strong T2 signals (Figure 1). Through T2WI, we observed a relatively strong overall signal and low center signal in 13 lesions. Fifteen lesions were also found to have an even low $\mathrm{T} 1$ signal and a mixed high and low T2 signal in the center. These lesions had small masses and were not clearly visible from the unenhanced MRI, however, cholangiectasis was present. Furthermore, 3 lesions were characterized as having a slightly high $\mathrm{T} 1$ and $\mathrm{T} 2$ evenness (Figure 2). The signals did not become significantly weaker with out-of-phase scanning. The detectable rate of T1WI combined with T2WI was $69.49 \%$ (41/59).
Symptoms on the dynamic contrast-enhanced MRI. All subjects $(\mathrm{n}=59)$ were given dynamic contrast-enhanced MRIs. $30(50.84 \%, 30 / 59)$ lesion peripheries in the arterial phase exhibited irregular ring or flower-ring enhancements. However, in the portal and delayed phases, the lesions were gradually filled by a contrast agent (Figure 3). The imaging characteristics of IMCC in the hepatobiliary phase were as follows: the tumor was surrounded with evidently round low signals and was significantly different from the liver parenchyma boundary. Low signal is defined as a round enhanced area of the tumor periphery during the arterial phase. Over time (60-90 mins), a delayed clear round low signal appeared. The tumor center was completely filled by a contrast agent and displayed high or mixed signals (mainly high signal). During the hepatobiliary phase, both a complete enhancement (Figure 4) and a patchy or latticed enhancement were seen. The lesion periphery had rich tumor cells and the lesion center was mainly made up of an interfibrillar and desmocyte substance. Simultaneously, 22 $(37.29 \%, 22 / 59)$ lesions exhibited an uneven mild/moderate patchy enhancement in the arterial phase and a progressive and concentric enhancement during the portal and delayed phases (Figures 4, 5). Among these 22 lesions $(37.29 \%, 22 / 59)$,
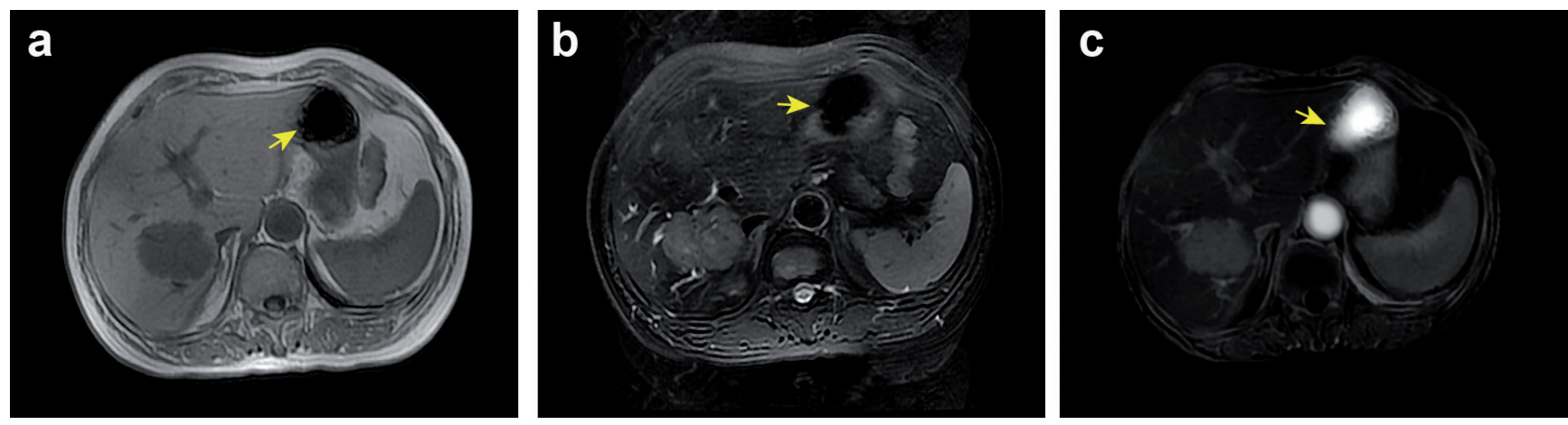

Figure 2. Lesion was located in the right anterior and left internal lobe of liver. a: evenness of slightly low signal on T1WI; b: mixture of slightly high signal and visually star or flake signal in center on T2WI; c: high signal in peripheral part but low signal in center on DWI (b=1200); DWI, diffusionweighted imaging. 

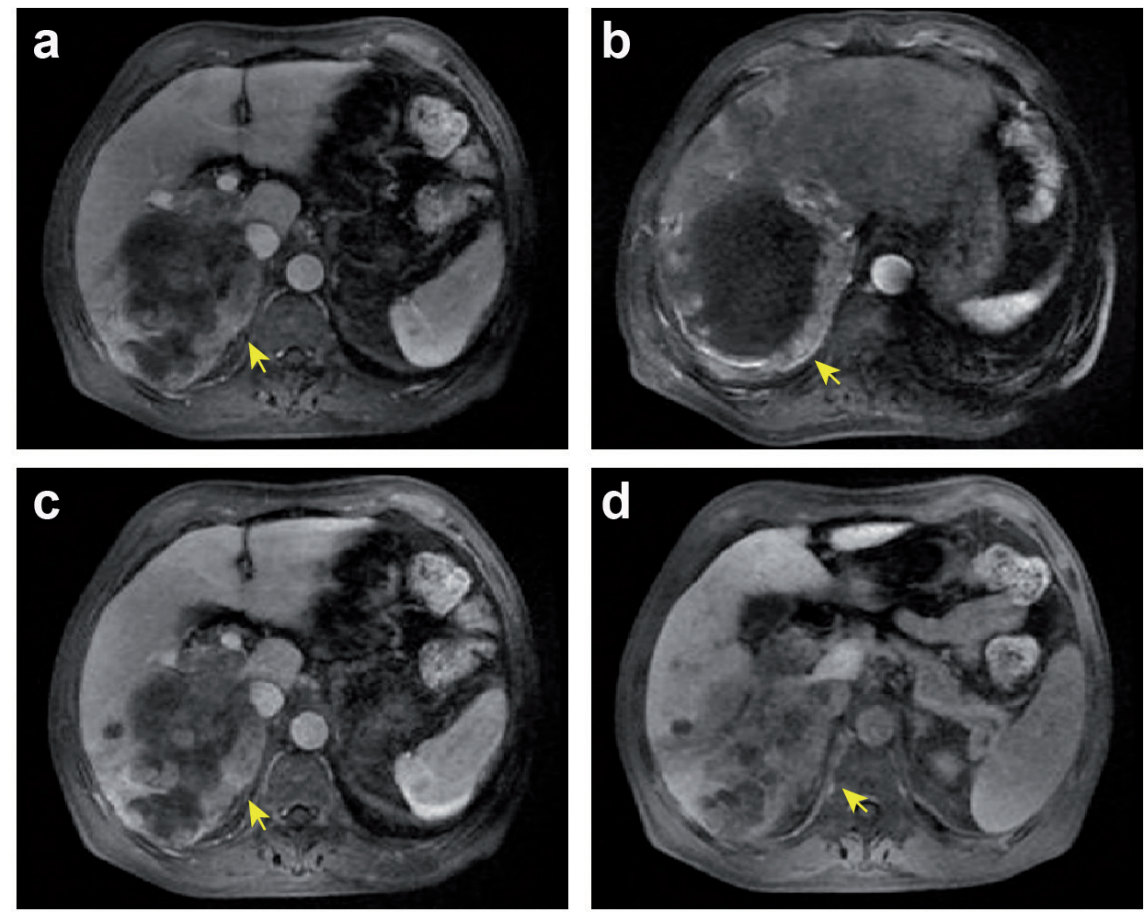

Figure 3. Lesion was located in the right posterior lobe of liver. a to d: the lesion with ring enhancement were gradually filled by contrast agent in portal and delayed phases.

8 had a decreased degree of enhancement early in the delayed phase. Five $(8.47 \%, 5 / 59)$ lesions emerged without any significant enhancement during the arterial phase and had a gradual enhancement in the delayed phase. The lesion peripheries had rich tumor cells and the lesion centers had significant necrosis, whereby island like tumor cell lesions were observed. The remaining $2(3.39 \%, 2 / 59)$ lesions had a relatively average enhancement, a decreased delayed scanning enhancement and had comparatively high signals. The lesion center had visible small spotted low signals, rich tumor cells and little interfibrillar
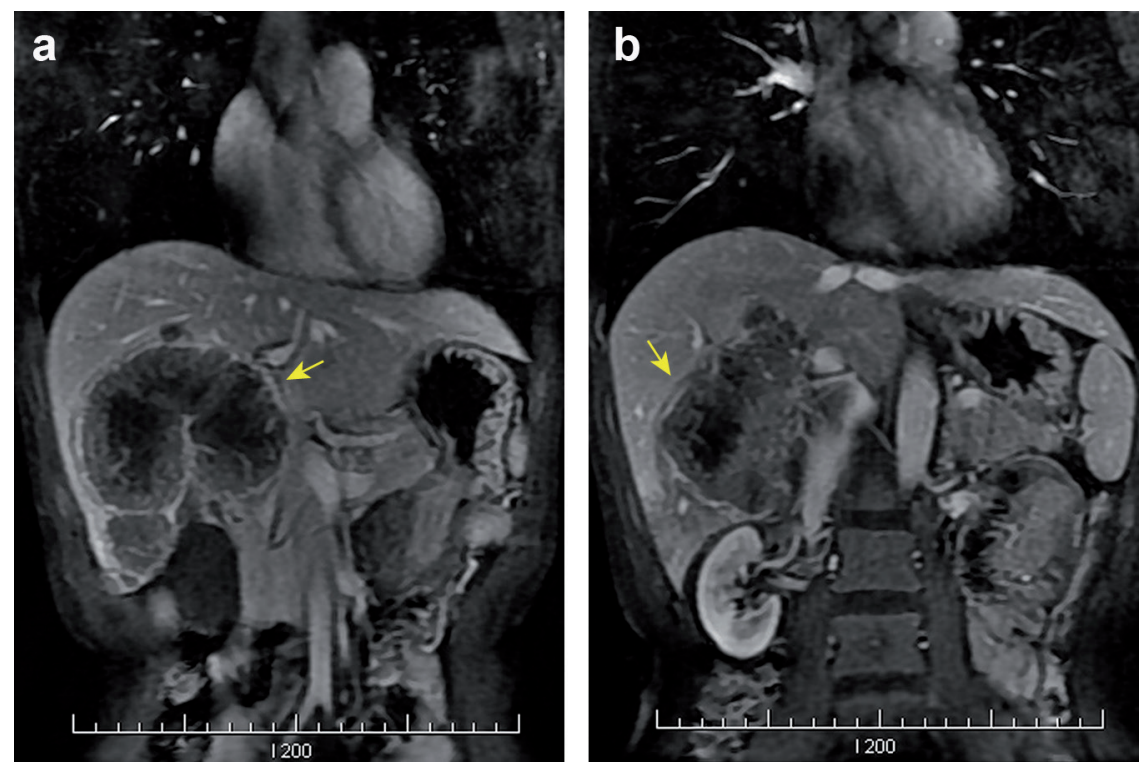

Figure 4. a: image at delayed $23 \mathrm{~min}$; lesion with clear edge presented low signal and lesion center failed to be completely filled; b: image in hepatobiliary phase at delayed $80 \mathrm{~min}$; lesion with clear edge presented round low signal and lesion center was completely filled. 

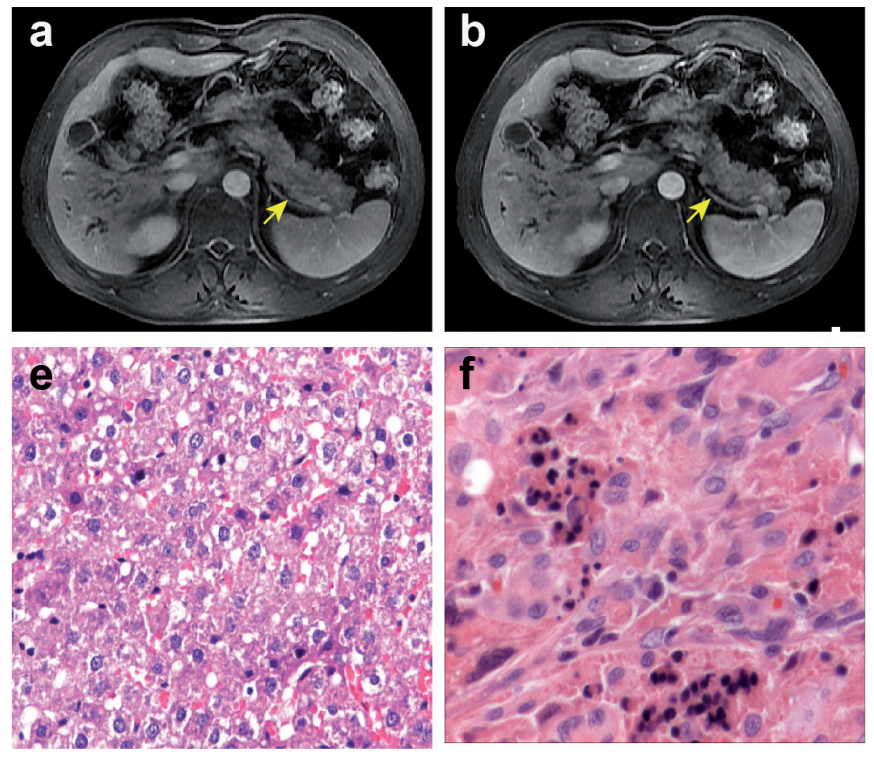

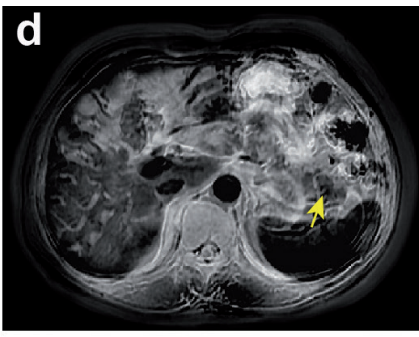

$\mathbf{h}_{\text {600 }}$ Time-signal intensity curve

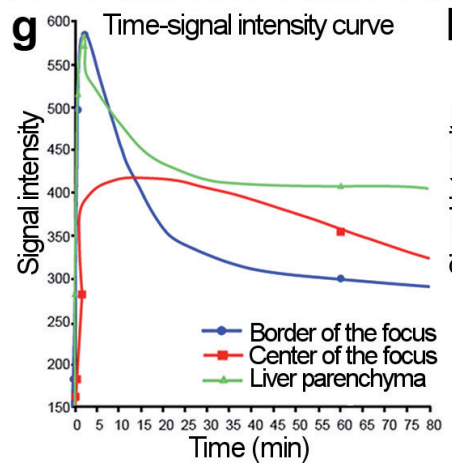

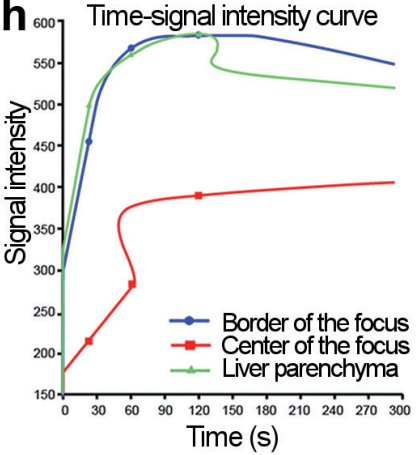

Figure 5. A 42-year-old IMCC man with lesion located across the left and right lobe of liver. a to c: the lesion periphery with patchy mild enhancement in early arterial phase and the enhancement gradually filled center; d: ADC signal declined in early enhancement; e and f: HE staining (400x); g: the time-signal intensity curve with interval time frame between 0 and hepatobiliary phase; h: the time-intensity curve with interval time frame between 0 and 5 minutes; ADC, apparent diffusion coefficient.

substances. A combination of the arterial and portal phases had a Gd-BOPTA-enhanced MRI detectable rate of 100\% (59/59).

Characteristics of the time-signal intensity curve from the lesion periphery, lesion center and liver parenchyma in
IMCC. The signal intensity of the lesion periphery increased rapidly during the early phase of dynamic contrast-enhancement. It reached a peak in the equilibrium phase and then declined (initially at a fast rate followed by a slower rate). The
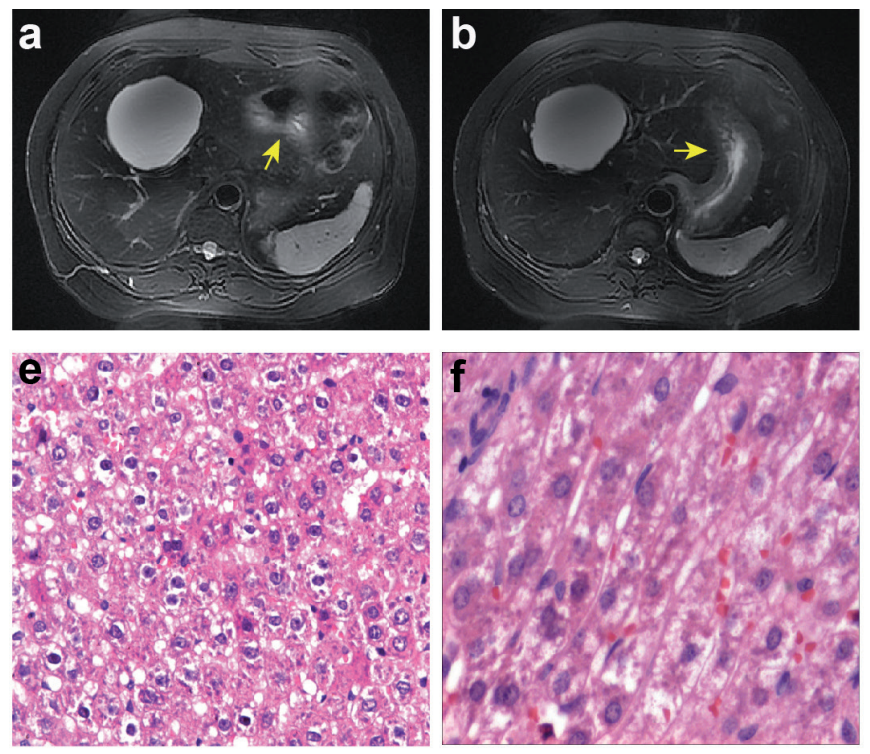
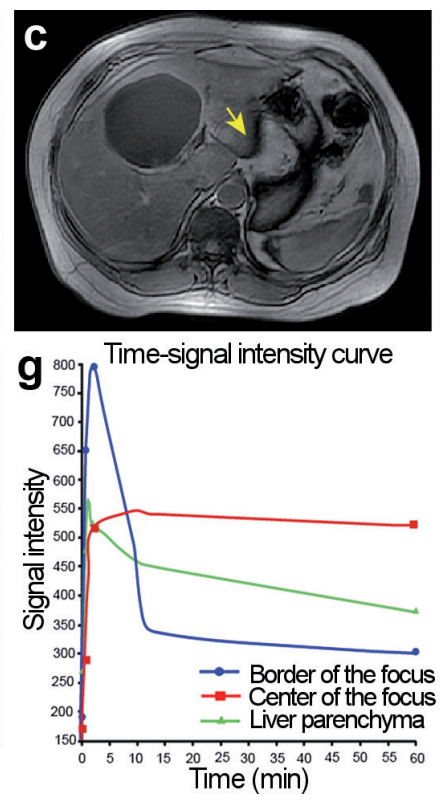

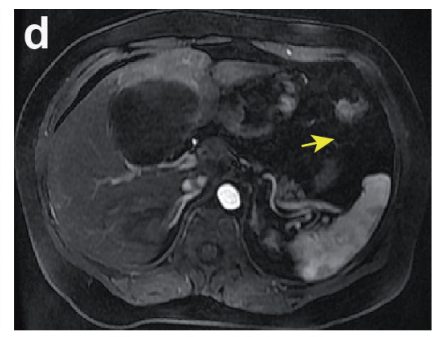

h ${ }^{800}$ Time-signal intensity curve

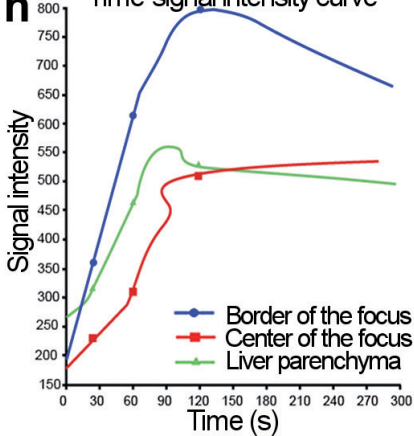

Figure 6. A 48-year-old IMCC woman with lesion located in the left lateral lobe of liver. a to c: the lesion periphery had patchy enhancement which gradually filled lesion center; d: ADC displayed low signal in the early enhancement; $\mathrm{e}$ and $\mathrm{f}$ : HE staining $(400 \times)$. g: the time-signal intensity curve with interval time frame between 0 and hepatobiliary phase; h: the time-intensity curve with interval time frame between 0 and 5 minutes; IMCC, intrahepatic mass-forming cholangiocarcinoma; ADC, apparent diffusion coefficient. 
signal intensity of the lesion center also showed a rapid elevation in the early period of dynamic contrast-enhancement. It reached a high value in the equilibrium phase (which was lower than that of the lesion periphery), continued to rise reaching a peak in the middle/late period of the delayed phase and then gradually decreased. Signal intensity of the liver parenchyma quickly rose to a peak early during dynamic contrast-enhancement and then slowly decreased or plateaued. During the equilibrium phase, there was no significant signal difference between the lesion periphery and center $(P>0.05)$. Signals of both the lesion periphery and center were higher than of the liver parenchyma (both $P<0.05$ ). The lesion periphery, lesion center and liver parenchyma signal intensities exhibited large enhancements and had significant differences in enhancement rate $(F=36.93, P<0.001)$ (Figures 5, 6). Furthermore, the liver parenchyma enhancement rate was lower than that of the lesion periphery $(241.27 \pm 70.16$ vs. $307.96 \pm 91.33)$ but higher than that of the lesion center $(241.27 \pm 70.16$ vs. 186.44 \pm 66.97) (both $P<0.05$ ).

ADC values of the lesion periphery, lesion center and liver parenchyma in IMCC. The DWI demonstrated high signal unevenness and in some cases the lesion peripheries had high signals and the lesion centers had relatively low signals (with irregular patches). The detectable rate of DWI was $100 \%$ (59/59). There were significant differences among the $\mathrm{ADC}$ values of the lesion periphery during early enhancement and the lesion center and liver parenchyma during delayed enhancement (all $P<0.001$ ). Furthermore, significant differences were also found when a pair-wise comparison was performed on these three ADC values (all $P<0.05$ ) (Table 2). At the same time, the DWI showed high signals during enhancement of the lesion periphery and reduced ADC values (Figures 3-6).

IMCC related signs. Forty patients $(67.80 \%, 40 / 59)$ had portal vein stenosis or occlusion resulting from IMCC invasion and tumors embedding to a nearby portal vein branch. And 16 patients $(27.12 \%, 16 / 59)$ had portal vein thrombosis and 37 patients $(62.71 \%, 37 / 59)$ had cholangiectasis around the tumors. In addition, 40 patients $(67.80 \%, 40 / 59)$ had stretched liver capsules around the tumors, 21 patients $(35.59 \%, 21 / 59)$ had liver lobes atrophy and 19 patients $(32.14 \%, 19 / 59)$ had sub-lesions around the tumors. Among the 22 (37.29\%, 22/59) patients with bile duct stones, 7 had gallstones, 2 had choledocholithiasis, 4 had bile duct stones in the right lobe,
2 had bile duct stones in the left lobe, 6 had bile duct stones in both lobes and 1 had bile duct stones, gallstones and choledocholithiasis in both lobes. In the same liver lobe, 18 patients $(81.82 \%, 18 / 22)$ emerged with both bile duct stones and IMCC. Furthermore, 38 patients $(64.41 \%, 38 / 59)$ had both IMCC and cholecystitis. A total of 33 patients $(55.93 \%, 33 / 59)$ displayed lymph node enlargement in the hepatoduodenal ligament $(\mathrm{n}=$ 15), retroperitoneum $(n=5)$ or both $(n=13)$.

Histopathology of IMCC. All the patients with IMCC were pathologically diagnosed with IMCC and had white or graywhite hard masses (which had a clear or unclear boundary and irregular soft areas) in the partial center. There were 5 IMCC patients with intratumoral hemorrhage whose tumor sections showed taupe. Under the microscope, the tumor consisted of tumor cells with different degrees of differentiation, a variable number of fiber textures and had obvious cell pleomorphism, disordered arrangements and irregular duct-like structure (Figures 4, 5).

Detectable rates of the unenhanced MRI, Gd-BOPTA-enhanced MRI and DWI for diagnosing IMCC. The detectable rate of T1WI combined with T2WI was $69.49 \%$ (41/59). The combined detectable rate of the arterial and portal phases of the Gd-BOPTA-enhanced MRI was 100\% (59/59). The detectable rate of the DWI was $100 \%(59 / 59)$. The results indicate that the detectable rates of the Gd-BOPTA-enhanced MRI and DWI were both significantly higher than that of the unenhanced MRI (both $P<0.05$ ).

\section{Discussion}

In our study, we retrospectively analyzed the Gd-BOPTAenhanced MRI findings of 59 IMCC patients with an emphasis on the hepatobiliary phase. The result obtained from the unenhanced MRI indicated that MRI presentation is strongly associated with the histopathology of IMCC. The tumor consisted of oncocyte, fiber texture, coagulative necrosis and mucoprotein. Thus, TIWI commonly exhibits a low signal, while T2WI has a slightly high signal during the unenhanced MRI. Additionally, the majority of signals featured unevenness due to various distributions. A percentage of these tumor constituents were also in different cases or regions. Ba-ssalamah et al. reported that in unenhanced T1-weighted MR images, the intrahepatic cholangiocellular has an iso to hypointense signal intensity compared to the normal liver. However, in T2-

Table 2. The ADC value of lesion periphery, lesion center and liver parenchyma $\left(\times 10^{-3} \mathrm{~mm}^{2} / \mathrm{s}\right)$

\begin{tabular}{lccccc}
\hline & $50 \mathrm{~s} / \mathrm{mm}^{2}$ & $100 \mathrm{~s} / \mathrm{mm} 2$ & $500 \mathrm{~s} / \mathrm{mm} 2$ & $800 \mathrm{~s} / \mathrm{mm} 2$ & $1200 \mathrm{~s} / \mathrm{mm} 2$ \\
\hline Lesion periphery & $2.64 \pm 1.03$ & $2.28 \pm 1.06$ & $1.99 \pm 0.35$ & $1.52 \pm 0.27$ & $0.99 \pm 0.17$ \\
Lesion center & $3.23 \pm 1.61^{\mathrm{a}}$ & $3.16 \pm 1.27^{\mathrm{a}}$ & $2.75 \pm 0.59^{\mathrm{a}}$ & $2.21 \pm 0.45^{\mathrm{a}}$ & $1.67 \pm 0.30^{\mathrm{a}}$ \\
Liver parenchyma & $1.99 \pm 0.99^{\mathrm{ab}}$ & $1.74 \pm 0.87^{\mathrm{ab}}$ & $1.64 \pm 0.37^{\mathrm{ab}}$ & $1.33 \pm 0.36^{\mathrm{ab}}$ & $1.41 \pm 0.17^{\mathrm{ab}}$ \\
$F$ & 14.7 & 26.03 & 93.83 & 93.72 & $<1.1$ \\
$P$ & $<0.0001$ & $<0.0001$ & $<0.0001$ & $<0.0001$ & $<0.0001$ \\
\hline
\end{tabular}

ADC, apparent diffusion coefficient; a, compared to lesion periphery, $P<0.05$; b, compared to lesion center, $P<0.05$ 
weighted images it has a remarkably increased signal intensity and is dependent on the fibrous and mucin content [14].

The results of the dynamic contrast-enhanced MRI suggested that peripheral enhancement with progressive and concentric filling of Gd-BOPTA during the portal and delayed phases is typical in IMCC patients. This is linked to rich lesion fibrosis which leads to a slow wash in and wash out of the contrast medium. Gd-BOPTA involves a combination of both extracellular and hepatobiliary distribution through two main temporal acquisition phases (dynamic and delayed) [15]. Approximately $3 \%$ to $5 \%$ of Gd-BOPTA is performed through functioning hepatocytes. These are discharged into the bile due to its hepatobiliary properties and strong performance during the dynamic phase liver imaging. This is a result of increased relativity compared to other extracellular gadolinium-based contrast agents and weak transient protein binding [10]. After the enhanced MRI, the peripheral enhancement exhibited a ring shape during the arterial phase due to the number of tumor epithelial cells which contain less fibrous connective tissue [16]. In our study, 91.5\% of IMCC patients experienced early peripheral enhancement and displayed an irregular ring or flower-ring shape during the arterial phase.

Interestingly, the lesion periphery signal intensity increased rapidly during dynamic contrast-enhancement, reached a peak during the equilibrium phase and then declined. This enhancement rate was greater than that of the lesion center and liver parenchyma. This suggests that during the process of extracellular intravascular Gd-BOPTA, the lesion periphery contained a large number of active tumor cells and blood vessels. Largely consistent with our results, Wang et al. indicated that hepatocellular cancer typically exhibits a strong tumor enhancement during the arterial phase and then loses much of its contrast in the later phases [17]. The dynamic enhancement patterns of focal liver lesions in the gadoxetate disodium-enhanced MRI may be different from those in the extracellular agent contrast-enhanced MRI [18]. The Gd-BOPTA-enhanced MRI can depict morphological changes in the biliary system. These pharmacokinetic properties of mangafodipir trisodium make an MRI the ideal contrast agent for biliary kinetic imaging. However, it is impossible to obtain dynamic enhanced imaging of the liver [19].

Additionally, our study calculated the ADC values of the lesion periphery, lesion center and liver parenchyma using a DW-MRI. DWI can be used to differentiate abnormal tissues from normal tissues and $\mathrm{ADC}$ is a quantitative parameter from DW-MR imaging which combines the influences of capillary perfusion and water diffusion in the extracellular extravascular space [20]. In our study, the ADC value of the lesion periphery was lower than that of both the lesion center and liver parenchyma. In addition, the enhancement section of the lesion center was largely made up of fiber tissues with only a few malignant cells. Therefore, its ADC value was higher than that of the liver parenchyma.

In conclusion, our study provides convincing evidence that both a Gd-BOPTA-enhanced MRI and DWI have higher detectable rates and accuracy than an unenhanced MRI. We also suggest that early enhancement of the lesion periphery during the hepatobiliary phase is decreased in the Gd-BOPTAenhanced MRI. Furthermore, DWI exhibits a high signal and has a reduced ADC value during the early enhancement of mass periphery. However, an unenhanced MRI has a better overall diagnostic performance in the detection of breast cancer and a better specificity for both DWI and STIR sequences [21]. This is not specific to just unenhanced-MRIs and in fact can appear in the CE-MRE technique. Some arteries could not be evaluated due to bad positioning of the $3 \mathrm{D}$ volume or thinness [22]. DWI is sensitive to the molecular diffusion of water in tissues, indexes such as perfusion, tissue disorganization, cellularity, extracellular space and other variables. However, it can be used to diagnose a wide range of diseases (except cervical and endometrial cancers), determine the extent of parametrical invasion and depth of myometrial invasion in GTD $[23,24]$. The presence of IMCC during the hepatobiliary phase plays an important role in the diagnosis and identification of constituents. This study was conducted retrospectively, therefore, the radiologists were already aware of the pathologic diagnosis and limitations in the clinical conditions and study samples exist. Comparison experiments which contain different enhanced agents of IMCC diagnosis have not been conducted. Comparison studies with larger sample sizes which focus on different enhanced agents are required to further evaluate the diagnostic significance of an unenhanced MRI, Gd-EOB-DTPA-enhanced MRI and DWI. Nevertheless, our study provides a new targeted therapy for treating IMCC. The use of Gd-BOPTA-enhanced MRIs and DWI may strengthen the diagnosis of MICC and help to differentiate MICC from other lesions in a timely manner.

Acknowledgements: We acknowledge the reviewers for their helpful comments on this paper.

\section{References}

1] GUGLIELMI A, RUZZENENTE A, CAMPAGNARO T, PACHERA S, VALDEGAMBERI A et al. Intrahepatic cholangiocarcinoma: prognostic factors after surgical resection. World J Surg 2009; 33: 1247-1254. https://doi.org/10.1007/ s00268-009-9970-0

[2] KIM SA, LEE JM, LEE KB, KIM SH, YOON SH et al. Intrahepatic mass-forming cholangiocarcinomas: enhancement patterns at multiphasic CT, with special emphasis on arterial enhancement pattern--correlation with clinicopathologic findings. Radiology 2011; 260: 148-157. https://doi. org/10.1148/radiol.11101777

[3] KANG Y, LEE JM, KIM SH, HAN JK, CHOI BI. Intrahepatic mass-forming cholangiocarcinoma: enhancement patterns on gadoxetic acid-enhanced MR images. Radiology 2012; 264: 751-760. https://doi.org/10.1148/radiol.12112308

[4] CHAITEERAKIJ R, YANG JD, HARMSEN WS, SLETTEDAHL SW, METTLER TA et al. Risk factors for intrahepatic 
cholangiocarcinoma: association between metformin use and reduced cancer risk. Hepatology 2013; 57: 648-655. https:// doi.org/10.1002/hep.26092

[5] SHIRABE K, MANO Y, TAKETOMI A, SOEJIMA Y, UCHIYAMA $H$ et al. Clinicopathological prognostic factors after hepatectomy for patients with mass-forming type intrahepatic cholangiocarcinoma: relevance of the lymphatic invasion index. Ann Surg Oncol 2010; 17: 1816-1822. https://doi. org/10.1245/s10434-010-0929-Z

[6] LUO X, YUAN L, WANG Y, GE R, SUN Y et al. Survival outcomes and prognostic factors of surgical therapy for all potentially resectable intrahepatic cholangiocarcinoma: a large single-center cohort study. J Gastrointest Surg 2014; 18: 562-572. https://doi.org/10.1007/s11605-013-2447-3

[7] SLATTERY JM, SAHANI DV. What is the current state-of-theart imaging for detection and staging of cholangiocarcinoma? Oncologist 2006; 11:913-922. https://doi.org/10.1634/theoncologist.11-8-913

[8] THIAN YL, RIDDELL AM, KOH DM. Liver-specific agents for contrast-enhanced MRI: role in oncological imaging. Cancer Imaging 2013; 13: 567-579. https://doi.org/10.1102/1470$\underline{7330.2013 .0050}$

[9] ZHAO X, HUANG M, ZHU Q, WANG T, LIU Q. The relationship between liver function and liver parenchymal contrast enhancement on Gd-BOPTA-enhanced MR imaging in the hepatocyte phase. Magn Reson Imaging 2015; 33: 768-773. https://doi.org/10.1016/j.mri.2015.03.006

[10] FRYDRYCHOWICZ A, LUBNER MG, BROWN JJ, MERKLE EM, NAGLE SK et al. Hepatobiliary MR imaging with gadolinium-based contrast agents. J Magn Reson Imaging 2012; 35: 492-511. https://doi.org/10.1002/jmri.22833

[11] GUPTA RT, ISEMAN CM, LEYENDECKER JR, SHYKNEVSKY I, MERKLE EM et al. Diagnosis of focal nodular hyperplasia with MRI: multicenter retrospective study comparing gadobenate dimeglumine to gadoxetate disodium. AJR Am J Roentgenol 2012; 199 35-43. https://doi.org/10.2214/ AJR.11.7757

[12] GUPTA RT, MARIN D, BOLL DT, HUSARIK DB, DAVIS $\mathrm{DE}$ et al. Hepatic hemangiomas: difference in enhancement pattern on $3 \mathrm{~T}$ MR imaging with gadobenate dimeglumine versus gadoxetate disodium. Eur J Radiol 2012; 81: 2457-2462. https://doi.org/10.1016/j.ejrad.2011.10.014

[13] M PN. World Medical Association publishes the Revised Declaration of Helsinki. Natl Med J India 2014; 27: 56.

[14] BA-SSALAMAH A, UFFMANN M, SAINI S, BASTATI N, HEROLD C et al. Clinical value of MRI liver-specific contrast agents: a tailored examination for a confident non-invasive diagnosis of focal liver lesions. Eur Radiol 2009; 19: 342-357. https://doi.org/10.1007/s00330-008-1172-X
[15] SCHNEIDER G, ALTMEYER K, KIRCHIN MA, SEIDEL R, GRAZIOLI L et al. Evaluation of a novel time-efficient protocol for gadobenate dimeglumine (Gd-BOPTA)-enhanced liver magnetic resonance imaging. Invest Radiol 2007; 42: 105-115. https://doi.org/10.1097/01.rli.0000251539.05400.06

[16] MASSELLI G, MANFREDI R, VECCHIOLI A, GUALDI G. MR imaging and MR cholangiopancreatography in the preoperative evaluation of hilar cholangiocarcinoma: correlation with surgical and pathologic findings. Eur Radiol 2008; 18: 2213-2221. https://doi.org/10.1007/s00330-008-1004-Z

[17] WANG L, VAN DEN BOS IC, HUSSAIN SM, PATTYNAMA PM, VOGEL MW et al. Post-processing of dynamic gadolinium-enhanced magnetic resonance imaging exams of the liver: explanation and potential clinical applications for color-coded qualitative and quantitative analysis. Acta Radiol 2008; 49: 6-18. https://doi.org/10.1080/02841850701630300

[18] JEONG HT1, KIM MJ, CHUNG YE, CHOI JY, PARK YN et al. Gadoxetate disodium-enhanced MRI of mass-forming intrahepatic cholangiocarcinomas: imaging-histologic correlation. AJR Am J Roentgenol 2013; 201: W603-611. https:// doi.org/10.2214/AJR.12.10262

[19] AKPINAR E, TURKBEY B, KARCAALTINCABA M, BALLI $\mathrm{O}, \mathrm{AKKAPULU} \mathrm{N}$ et al. Initial experience on utility of gadobenate dimeglumine (Gd-BOPTA) enhanced T1-weighted MR cholangiography in diagnosis of acute cholecystitis. J Magn Reson Imaging 2009; 30: 578-585. https://doi.org/10.1002/ jmri.21887

[20] SIJENS PE. Parametric exploration of the liver by magnetic resonance methods. Eur Radiol 2009; 19: 2594-2607. https:// doi.org/10.1007/s00330-009-1470-y

[21] BELLI P, BUFI E, BONATESTA A, PATROLECCO F, PADOVANO $\mathrm{F}$ et al. Unenhanced breast magnetic resonance imaging: detection of breast cancer. Eur Rev Med Pharmacol Sci 2016; 20: 4220-4229.

[22] SEBASTIA C, SOTOMAYOR AD, PANO B, SALVADOR R, BURREL $M$ et al. Accuracy of unenhanced magnetic resonance angiography for the assessment of renal artery stenosis. Eur J Radiol Open. 2016; 3: 200-206. https://doi.org/10.1016/j. ejro.2016.07.003

[23] WAN Q, DENG YS, ZHOU JX, YU YD, BAO YY et al. Intravoxel incoherent motion diffusion-weighted MR imaging in assessing and characterizing solitary pulmonary lesions. Sci Rep 2017; 7: 43257. https://doi.org/10.1038/srep43257

[24] SEFIDBAKHT S, HOSSEINI F, BIJAN B, HAMEDI B, AZIZI T. Qualitative and quantitative analysis of diffusion-weighted imaging of gestational trophoblastic disease: Can it predict progression of molar pregnancy to persistent form of disease? Eur J Radiol 2017; 88: 71-76. d https://doi.org/10.1016/j. ejrad.2016.12.036 